\title{
COAL SCIENCE
}

T HE biennial International Conferences on Coal Science, which began in The Netherlands in 1955 , moved on the most recent occasion to France: the fourth Conference was held at Le Touquet during May 30-June 2. Tradition was followed in the extent of local co-operation, and the Mayor, Dr. Jules Pouget, received delegates and their wives at the Town Hall. A tribute should be paid, for the excellent organization and remarkable hospitality, to the French sponsors, the Centre d'Études et Recherches des Charbonnages de France: particularly to Prof. M. Letort (a director-general of the Centre), who presided, R. Loison, the French member of the International Committee, and A. Ploix, who undertook all the details of organization. The conference was fortunate in gaining Mr. Berkx's experience from the previous meeting which he organized in The Netherlands.

Interest continues to increase; the recent Conference included a delegation from the U.S.S.R., and Spain, Brazil and Czechoslovakia were represented. On the other hand, delegates from India and East Germany, who attended in 1959, were absent. The papers presented originated from ten countries. Titles of more than one hundred were submitted, and this number was only reduced with some difficulty to about 50 by narrowing the field-to papers of direct relevance to the chemistry, physics and carbonization of the coal substance.

Since authors are submitting their papers to journals of their choice and proceedings of the Conference will not be published, the 53 papers presented will all be mentioned briefly, grouped under the three main headings used in the sessions. An independent summary by A. Ladam in the French language will be found in a late-1961 issue of Chimie et Industrie. A numbered list of references so far available has been appended.

\section{Chemical Properties}

P. H. Given ${ }^{1}$ reviewed his proposed molecular model for coals in the light of more recent work on dehydrogenation, nuclear magnetic resonance, and other evidence; he concluded that the general features of the model had been confirmed, but indicated changes in structural detail that appeared desirable.

H. A. G. Chermin attempted to deduce from measured densities and elementary analyses the mean size and proportion of aromatic units in coals, and directed special attention to the accuracy possible. Corrections to previous attempts were made and quite good agreement with X-ray measurements of mean size was found.

H. S. Rao, P. L. Gupta, F. Kaiser and A. Lahiri considered the assignment of the band at $1,600 \mathrm{~cm} .^{-1}$ in the spectra of coals. This band could have one or all of several origins; and the results of reducing the coals with lithium aluminium hydride, since this did not cause a change in intensity in the band, were considered to discount assignment to a carbonyl vibration. On a related subject was an investigation, by L. Czerski and L. Czuchajowski, of the same spectral band in polycondensates of $o_{-}, m^{-}$and $p$-dihydroxybenzenes with formaldehyde. When the para-derivatives were oxidized with ferric sulphate the $1,600 \mathrm{~cm} .^{-1}$ band increased in intensity, possibly owing to formation of hydroxyl/carbonyl chelate structures, supporting earlier assignments of this band in coals to such structures.

M. T. Monaghan and K. Littlewood attempted to use trimethyl-acetoaminohydrazide hydrochloride (Girard's reagent $T$ ) to extract carbonyl-containing substances from coals as water-soluble derivatives (see p. 128 of this issue of Nature). M. Weclewska described the effect on eight coals of exposing them to a variety of organic vapours. Conclusions were drawn as to the variation with rank of free and hydro. gen-bonded hydroxyl groups. P. H. Given, M. E. Peover and W. F. Wyss had examined the chemical reactions and properties of some petrological constituents of coals. Moderate differences in hydroxyl content and reducibility were found, the general molecular pattern being similar for all the macerals studied. B. K. Mazundar, S. Ganguly, N. Gopal De and $A$. Lahiri had investigated the effect of phosphoric and sulphuric acids on coal structure; they found that a constant proportion of the total carbon in the coal (92 per cent) was retained in the chars produced when the products were carbonized. Since extensive dehydrogenation did not result, an apparent condensation between the alicyclic structures and the remainder of the molecule was postulated to explain the inhibition of tar formation caused by the treatment. J. D. Brooks and T. M. Spotswood ${ }^{2}$ discussed the reaction of carbonized coals with bromine and with sodium in various forms; the results were consistent with the presence of polycyclic hydrocarbon structures.

Several papers were concerned with oxidation or oxidation products. H. E. J. Brusset compared humic acids extracted from low-rank coals with those produced synthetically from polyphenols by oxida. tion; he adduced support for an analogy between these classes of material. Oxidation of coal in alkaline medium at $260^{\circ}$ C. was described by J. E. Germain, J. Guillon and P. Delattre. The high yields of benzene carboxylic acids obtained were extracted with methyl ethyl ketone and subjected to chromatography. Fractions were characterized according to the number of carboxyl groups per benzene ring; increase in rank of coal was accompanied by a decrease in the mean number of groups per ring (about 4) and an increase in total yield of these acids. G. J. Lawson and S. G. Ward reported a further examination of the resolution of sub-humic acids (secondary oxidation products of coal) partly by means of ion-exchange resins. Several known acids were isolated, including some previously reported (for example, glycollic and mellitic acids) and two aliphatic acids containing nitrogen (nitrilotriacetic acid and oxalylglycine). B. Jüttner ${ }^{3}$ described the oxidation of coke with nitric acid followed by sodium hypochlorite and extraction with butanone. The yield of mellitic acid increased sharply between $600^{\circ}$ and $700^{\circ} \mathrm{C}$. carbonization temperature, and then fell slightly. A related topic was a description of the use of ozonolysis in research on coal tar, by P. G. Copeland. Oxidized pitch fractions and model substances were further decomposed with alkaline peroxide and/or permanganate in pyridine and the products were identified. Mechanisms were established from the work with 
models, but the products from coal tar pitch had not yet been identified.

Reaction of coals with alkaline solution at $200^{\circ}$ $300^{\circ} \mathrm{C}$. was found, by M. Ihnatowicz $z^{4}$, in many cases to increase greatly the agglutinating values observed when the products were later heated. A strong hydrogenating action accompanying hydrolysis was indicated.

Possibly the most novel work described was that of L. A. Herédy and M. B. Neuworth on a depolymerization of bituminous coal at only $100^{\circ} \mathrm{C}$. The coal was allowed to react with a labelled phenol-boron trifluoride system; depolymerization was recognized by substantially increased solubility in phenol. Models were used to elucidate the reactions taking place. With coal, cleavage of aromatic-aliphatic linkages and also interchange of aromatic units with phenol were both involved. Some of the structural units were thus characterized in terms of analysis and molecular weight, and evidence of bridges other than via methylene groups was obtained; possible model structures for coal that would fit the evidence obtained were discussed. E. E. Donath adduced the presence of polynuclear aromatic units in pitches from coal hydrogenation from a study of the ease of change in hydrogen content.

Two papers considered processes that may occur during the formation of coals. G. Huck, J. Karweil and $\mathrm{K}$. Patteisky ${ }^{5}$ showed in an experimental study that only dynamic pressure is likely to accelerate increase in rank, and that spores are unsuitable as indicators of the temperature of formation. I. A. Breger considered the 'coalification' of wood in uranium-bearing sandstones. It seems that nuclear radiation has caused dehydrogenation and condensation, leading to effects similar to those produced by biochemical coalification.

P. Dath, H. Renson and E. H. Grand'ry reported work on the influence of rank and petrological composition on the behaviour of coals in the AudibertArnu dilatometer and came to the conclusion that both are important. A. Ihnatowicz, M. Lason and M. Zyla had shown by treatment of coal with alcoholic potash and with pyridine, together with hydroxyl determinations and adsorption measurements, that the two treating agents used altered these properties in a similar manner. Evidence that this was due to a mechanical loosening of the structure was obtained. J. D. Brooks, J. F. Stephens and H. Silberman ${ }^{6}$ had examined the $\mathrm{X}$-ray diffraction patterns and functional groups in chars from carbonized salts of brown coal. The 002 band was shifted, and it was suggested that in brown coals this band may possibly arise from an interchain $d$-spacing. Phenolic groups in the salt form were less-easily eliminated by heating than in the untreated coals. B. Roga surveyed Polish and international classifications of cosls and suggested modifications.

\section{Physical Properties}

S. Ergun and M. Mentser carried out investigations on the X-ray scattering patterns of ten anthracites and meta-anthracites. Those of higher rank gave rise to three-dimensional reflexions, indicating that coal cen graphitize during metamorphism. Nuclear magnetic resonance techniques were applied by $J$. Smidt, W. van Raaijen and D. W. van Krevelen to show that the mobility of the protons in coals changes with rank, and that it shows a characteristic variation with temperature for the coking vitrinites. The effect, on earlier published conclusions, of this variation was discussed. Pitches were also studied. M. Jacubowicz and J. Uebersfeld described results obtained with several coals when studied by double magnetic resonance. The nature of the interactions (dipolar, etc.) between the electronic and the nuclear magnetic moments was determined, and a better understanding was achieved of the electron spin resonance ascribed to free radicals. Some possibilities for studying adsorption were also mentioned.

Two papers were concerned with the specific surface and heat of wetting of coals and carbonized coals. S. Prégermain, S. Durif and P. Chiche studied the adsorption of water, methanol and nitrogen on carbonized coals, and their low-angle $X$-ray scattering. Inter alia, a relation was found between the ease of graphitization and the development of the internal surface. L. Robert and S. Prégermain found a quantitative relation between heat of wetting in methanol, per unit surface of the carbonaceous solid, and its oxygen content; extrapolated to zero oxygen content the heat evolved is still considerable, and the chief effect, therefore, is to raise the estimated surface areas of high-rank coals and chars while leaving those of low-rank coals little changed. J. W. Phillips reported on the self-adhesion of coal during briquetting without a binder. The physical properties, and the structure revealed by photomicrographs, of the briquettes were linked with the stress and strain relations during the application of pressure with additional shear strain. D. H. Everett, E. Redman, A. J. Miles and D. H. Davies reported adsorption isotherms on chars made from polyvinylidene chloride. Important differences in hysteresis, relevant to the mechanism of coal carbonization, were discussed.

Reflectances of vitrinites carbonized at a series of pressures were reported by M.-Th. Mackowsky. An attempt was made to relate the changes found with the chemical changes known to occur during heating. L. A. Gilbert had determined refractive indices and absorption coefficients of polished surfaces of coals, in the ultra-violet region, by a polarized light method. This was the first application of this method to coals and yielded valuable results. All the vitrains used showed a strong aromatic-type absorption around 2600 A. S. Ergun and J. T. McCartney reported the refractive indices of ultra-thin sections of coals and graphite in the visible region and extinction coefficients in the ultra-violet and visible regions. The results were tentatively considered to lead to conclusions regarding the aromatic units in coals similar to those derived from $\mathrm{X}$-ray investigations.

E. de Ruiter, R. Leutner and H. Tschamler reported the electrical resistance of powdered coals under pressure and of their products of extraction by pyridine in relation to temperature. Little change in the energy of electronic activation for coals was observed between 80 and 90 per cent carbon content; values of this energy were similar for the residues of extraction of solvent, but greater for the soluble products by extraction. The results for the extracts were considered to accord with conclusions from X-ray diffraction.

\section{Thermal Decomposition}

No less than four papers were concerned with the plastic softening of coking coals in the early stages of heating. Rheological properties during the initial softening had been studied by $H$. R. Brown and P. L. Waters ${ }^{8}$. They found that the temperatures of 
initial softening of a wide range of Australian coals were almost independent of rank within the range of 82-89 per cent carbon content, and that the softening was initially reversible; but maximum fluidity and solidification temperature were dependent on rank. A medium-rank coal behaved as though the flow were Newtonian until the onset of active decomposition. A mean activation energy of about $70 \mathrm{kcal} . / \mathrm{mole}$ was estimated. J. C. Macrae and K. S. Murthi ${ }^{9}$ had determined, in a special cone viscometer, the variations of fluidity at a constant temperature below the active decomposition point; they interpreted these in relation to the microscopically observed porosity of the char after cooling and the amount of chloroform extract obtainable from this char. The duration and magnitude of fluidity were found to be highly dependent on temperature, and the increasing fluidity before the maximum to be directly related to the dilation of the mass with gas bubbles; the amount of chloroform-soluble material present did not change materially over the same period and therefore was considered unlikely to produce this increase.

I. G. C. Dryden and W. K. Joy ${ }^{10}$ had investigated the provenance of the fusible chloroform-soluble material in heated coals, by comparing similar materials obtained in three independent ways. They concluded that this fusible material is partly present in the unheated coal and partly formed or disengaged during heating, the proportion originally present increasing with increase of rank up to 100 per cent in prime coking coals. Evidence was also presented leading to the conclusion that the portion originally present is also more resistant than the remainder to thermal decomposition. The plasticity developed was considered, on limited evidence, to depend critically on the concentration of fusible materialnot entirely in agreement with the conclusions of Macrae and Murthi. A. D. Dainton demonstrated by static beam-bending methods that visco-elastic behaviour persisted in the semi-coke from a coking coal above the commonly accepted point of solidification for the plastic coal, that is between $480^{\circ}$ and $800^{\circ} \mathrm{C}$.

Development of the fissures in coke that result from shrinkage, following resolidification of the plastic mass, was studied by $\mathbf{H}$. E. Blayden, H. C. Wilkinson and G. E. Oatley in relation to the temperature distribution in an experimental coke oven. A clear correlation between shrinkage and fissuring was observed, and these authors also concluded that semicokes may show visco-elastic behaviour. G. $H$. Taylor ${ }^{11} x \in$ ported the appearance in both reflected and transmitted light of coals at various stages in carbonization. Colour photomicrography was an important feature of the techniques used. Development of an optical mosaic structure from the plastic vitrinite was observed. The behaviour suggested that the resolidification of coke is analogous to a crystallization process. H. Pichler and D. H. Lee ${ }^{12}$ reported the effects of extracting a bituminous coal for various times with tetrahydrofuran. The consequent changes in plastic behaviour, dilatometer curves, volatile matter and other characteristics were described. W. F. Landers and J. B. Goodman had investigated the effects of temperature and gas pressure on the tar obtained from a lignite. Increased susceptibility to decomposition of the tar liberated between $500^{\circ}$ and $600^{\circ} \mathrm{C}$. was evident, and this was further increased by pressure.

S. Cernič reported an investigation, with the help of labelled pyrite, of the behaviour of organic and inorganic sulphur-containing constituents of a series of coals during carbonization. Evidence was obtained that inorganic and organic forms of sulphur were interchanged to some extent, according to the rank of coal, during the thermal decomposition. The effectiveness of carbonizing in hydrogen, ammonia and pre-heated steam, in reducing the sulphur content of the resulting coke or char, was also investigated; steam proved the best.

Heats of coking formed the subject of three papers; the definition used must be considered when making comparisons. D. Fitzgerald reported coking heats for an anthracite and several low-rank coals. An isothermal fluid bed calorimeter was used for the measurements. The poor agreement between results of other work was critically discussed. C. Meltzheim, J. Lahouste and A. Boyer had made a direct comparison of two methods of measuring the heats of carbonization, and found them in satisfactory agreement. The net heat of carbonization over the whole range of temperature, for a number of coals, was always exothermic, the value varying with rank in a complex manner in the region of 30-170 $( \pm 40)$ cal./gm. C. Kröger and J. J. Das ${ }^{13}$ had investigated the net heats of carbonization of individual macerals by direct calorimetry. The heats were not directly related to the yields of volatile products. A relation between the decomposition heat up to $500^{\circ} \mathrm{C}$. and the hydrogen/oxygen ratio and the carbonyl content of the coals investigated was, however, found. At $1,000^{\circ} \mathrm{C}$. vitrinite appeared to give the lowest heat values of the three macerals studied.

The rate of evolution of volatile matter from coal, during carbonization in a fluid bed with sand as heating medium, was interpreted by G. J. Pitt on the assumption that the coal is a complex mixture, each component of which decomposes independently by a first-order reaction. He showed that this assumption can lead to the linear change with logarithm of time observed, and with this assumption deduced from the results a distribution of activation energies (35-75 $\mathrm{kcal}$./mole, maximum at 50-55 kcal./mole).

A continued investigation of the pyrolysis of model compounds believed to resemble coal was reported by D. W. van Krevelen and H. W. den Hartog ${ }^{14}$. Polycondensates in which aromatic and non-aromatic structures (or oxygen atoms) alternated, prepared with labelled formaldehyde, were pyrolysed; from the weight, and analysis of and distribution of radioactivity in the products, conclusions were drawn about the influence of structure on the degree of (temperature) overlap in tar and gas evolution and on the weight loss-temperature curves; and it was concluded that $\mathrm{C}-\mathrm{O}$ bonds decomposed more readily than $\mathrm{C}-\mathrm{C}$ bonds.

In the next group of four papers, the work described had been directed towards the establishment of relations between volatile products of the pyrolysis of coal and the chemical structure of the coal itself. G. Claxton described work on the genesis of aromatic hydrocarbons using gas-liquid chromatography. In a coke oven, the peak evolution of non-aromatic hydrocarbons occurred earlier in the heating process than the peak for benzene formation; significant effects of the heating-rate were observed. In laboratory experiments more detailed information was obtained. A. Ladam, R. Ferrand, P. Payen and A. Boyer had studied the constituents of lowtemperature tars $\left(550^{\circ}\right.$ C.) from vitrinite and exinite concentrates, using vapour phase chromatography. Normal paraffins and olefines arose almost entirely 
from exinite, and the results suggested that as much as 10 per cent of the exinite used may consist of chains with about 30 carbon atoms; correspondingly, aromatic compounds from exinite tar were more highly alkyl-substituted than those from vitrinite tar. W. Waddington had compared the relative abundance of isomeric forms in tar with that to be expected from the postulated thermal reactions of a proposed structural model. He suggested that the results obtainod support the view that coals contain in part a 6 alkyl-4 propyl-2 methoxy phenol type of structure; this may be gradually converted to other structures by thermal/pressure reactions during coalification. C. Kröger and R. Brücker ${ }^{15}$ reported results from high-vacuum pyrolysis of coals and discussed these in relation to the coal structure. Products in the range $300^{\circ}-575^{\circ} \mathrm{C}$. were examined by gas chromatography and other methods. Rate constants and activation energies for the evolution of hydrogen, many simple hydrocarbons and the carbon oxides were estimated; and from these in turn, by means of allocation of reactions to structural elements on certain assumptions, quantitative parameters for 17 elements of structure, such as the proportion of aromatic carbon in the coal, were deduced.
There proved to be a shortage of time for formal discussion, and on the other hand many delegates remarked on the value of spontaneous extra-mural discussions. Further experiment is clearly necessary in an attempt to correct this lack of balance. It is hoped that, since these conferences continue to produce more novel material than the average delegate can comfortably digest, further innovations will be made at the next conference, to be held at Cheltenham during 1963.

I. G. C. DRYDEN

${ }^{1}$ Given, P. H., Fuel, 40, 427 (1961).

${ }^{2}$ Brooks, J. D., and Spotswood, T. M., Proc. Fifth Bien. Carb. Conf. U.S.A. (1961).

3 Jüttner, B., Brennstoff-Chem. (in the press).

' Thnatowicz, M., Prace Glónonego Instytutu Górnictwa (in the press).

'Huck, G., Karweil, J., and Patteisky, K., Brennstoff-Chem. (in the press); Freiberger Forschungsheften, A.229 (1061).

- Brooks, J. D., Stephens, J. F., and Silberman, H., Proc. Fifth Bien. Carb. Conf., U.S.A. (1961).

7 Ergun, S., and McCartney, J. T., Fuel, 40, 109 (1961).

8 Brown, H. R., and Waters, P. L., Fuel (in the press).

- Macrae, J. C., and Murthi, K. S., Fuel (in the press).

${ }^{10}$ Dryden, I. G. C., and Joy, W. K., Fuel (in the press).

11 Taylor, G. H., Fuel (in the press).

12 Pichler, H., and Lee, D. H., Brennstoff-Chem., 42, 241 (1961).

${ }^{13}$ Kröger, C., and Das, J. J., Brennstoff-Chem., 42, 223 (1961).

${ }^{4}$ Van Krevelen, D. W., and den Hartog, H. W., Brennstoff-Chem. (in the press).

${ }^{16}$ Krōger, C., and̉ Brüeker, R., Brennstoff-Chem., 42, 245 (1961).

\section{BIOCHEMICAL ENGINEERING}

\begin{abstract}
A SYMPOSIUM on "Biochemical Engineering" was convened by the Institution of Chemical Engineers on May 30 ; it is doubtful whether any two of the participants, lucid as they were on their own topies, would have agreed on a definition of the title. Though, on one hand, one may assume unanimity of outlook in chemical engineers, the term 'biochemist' has been used by many disciplines, from the quantitative biologist to the organic chemist by way of the medicel analyst. Moreover, in the fields where engineers encounter Nature in the raw, ought not such a title to be amplified to 'biological chemical engineering'? It is easy to see how confusing such exercises in labelling can be, though there is always a stimulation of interest and research by collecting certain topics together under a high-sounding title, however arbitrary.

Nevertheless, many industries are now based on the controlled use of biological processes. (Strangely enough, the largest industry of this type, brewing, was already developed more or less to its present size before the terms 'biochemist' or 'chemical engineer' existed.) It was the production of antibiotics by fermentation, especially under the high pressure of the second World War, that brought a variety of biological interests (botanists, zoologists, pharmacists, agriculturists, entomologists-for there were very few true biochemists, by any definition, available at the time) face to face with the engineer (rarely of the 'chemical' variety). The difficulties of communication were considerable and were overcome the hard way. It was also realized that similar problems existed in the food preservation industries and the sewage and effluent disposal services. To help with these problems, the establishment of courses in 'biochemical engineering' were proposed-seldom, however, with any clear idea of what the graduates should do afterwards, although the vague idea of a superman who could control both sides of the
\end{abstract}

industrial scene may have been in the minds of some proponents. The problerns were considerable, for whereas most of the engineers' deficiencies were conceptual and philosophical, most of the biologists' were factual and mathematical. Making good the former must have seemed easier or, perhaps, they were not so apparent and the new discipline became, at least for a time, the protégé of chemical engineering.

Two difficulties then faced the new dispensation ; first, that with normal chemical engineers at a premium, the supply of new graduate output could not hope for many years to affect the position ; second, that with the enormous rate of growth of each parent topic, no person could hope to keep up to date in both biochemistry and chemical engineering. It was clear, therefore, that for many years to ing the two functions of engineer and biologist would have to be realized by specialists in each discipline working side by side as separate entities. It was with this aim of bringing together the two groups that this symposium was called.

In the opening paper, Dr. D. J. D. Hockenhull reviewed the formal aspects of the production of economically desirable products from micro-organisms. He stated, as an axiom, that the primary function of a living organism is to reproduce itself. It does this by a complex interlinked system of biochemical reactions on the nutrients supplied to it. Interference with these processes may lead to the accumulation of intermediates or of alternative products derived from them. Industrially important examples of such interference were : unbalanced nutrition (acetic acid), deficient aeration (alcohol), mineral deficiency (citric acid), use of trapping agent (glycerol) or of foreign precursors (penicillin) or inborn error of metabolism (lysine). It was pointed out that these 'primary colours' of metabolic interference, in Dr. G. L. Solomon's words, were most usually applied in combination to-day to obtain the best results. A more 